\title{
Cardiac PET/CT with Rb-82: optimization of image acquisition and reconstruction parameters
}

\author{
P. Chilra ${ }^{1,3,4}$, S. Gnesin ${ }^{2,3}$, G. Allenbach ${ }^{3}$, M. Monteiro ${ }^{3}$, J. O. Prior ${ }^{3}$, L. Vieira ${ }^{4,5}$ and J. A. Pires Jorge ${ }^{1 *}$
}

\author{
* Correspondence: \\ jose.jorge@hesav.ch \\ ${ }^{1}$ Haute École de Santé Vaud - Filière \\ TRM, University of Applied Sciences \\ and Arts Western Switzerland, \\ Lausanne, Switzerland \\ Full list of author information is \\ available at the end of the article
}

\begin{abstract}
Background: Our aim was to characterize the influence of time-of-flight (TOF) and point spread function (PSF) recovery corrections, as well as ordered subset expectation maximization (OSEM) reconstruction parameters, in ${ }^{82} \mathrm{Rb} \mathrm{PET} / \mathrm{CT}$ quantification of myocardial blood flow (MBF) and myocardial flow reserve (MFR).

Rest and stress list-mode dynamic ${ }^{82}$ Rb PET acquisition data from 10 patients without myocardial flow defects and 10 patients with myocardial blood flow defects were reconstructed retrospectively. OSEM reconstructions were performed with Gaussian filters of 4,6 , and $8 \mathrm{~mm}$, different iterations, and subset numbers $(2 \times 24 ; 2 \times 16 ; 3 \times 16 ; 4 \times 16)$. Rest and stress global, regional, and segmental MBF and MFR were computed from time activity curves with FlowQuant ${ }^{\odot}$ software. Left ventricular segmentation using the 17segment American Heart Association model was obtained.
\end{abstract}

Results: Whole left ventricle (LV) MBF at rest and stress were $0.97 \pm 0.30$ and $2.30 \pm$ $1.00 \mathrm{~mL} / \mathrm{min} / \mathrm{g}$, respectively, and MFR was $2.40 \pm 1.13$. Concordance was excellent and all reconstruction parameters had no significant impact on MBF, except for the exclusion of TOF which led to significantly decreased concordance in rest and stress MBF in patients with or without perfusion defects on a coronary artery basis and in MFR in patients with perfusion defects.

Conclusions: Changes in reconstruction parameters in perfusion ${ }^{82} \mathrm{Rb}$ PET/CT studies influence quantitative MBF analysis. The inclusion of TOF information in the tomographic reconstructions had significant impact in MBF quantification.

Keywords: Rb-82, Myocardial blood flow, Myocardial flow reserve, Cardiac PET/CT reconstruction

\section{Background}

Hybrid cardiac positron emission tomography/computed tomography (PET/CT) with ${ }^{82}$ rubidium $\left({ }^{82} \mathrm{Rb}\right)$ is a useful clinical tool for the diagnosis of unknown or suspected coronary arterial disease $(\mathrm{CAD})$ and microvascular disease, improving the understanding of the pathophysiology of disease processes thus directly impacting the therapeutic approach [1-3]. This short-lived radioisotope (half-life 76.4 s) is a monovalent cationic analog of potassium produced by a ${ }^{82}$ strontium $/{ }^{82}$ rubidium $\left({ }^{82} \mathrm{Sr} /{ }^{82} \mathrm{Rb}\right)$ generator, allowing clinical imaging with short protocols $(20-30 \mathrm{~min}$ in total), providing better results than conventional SPECT techniques with improved values of sensitivity and specificity in the order of 90\% [4-6]. The main reasons for improved diagnostic

(c) The Author(s). 2017 Open Access This article is distributed under the terms of the Creative Commons Attribution 4.0 International License (http://creativecommons.org/licenses/by/4.0/), which permits unrestricted use, distribution, and reproduction in any medium, provided you give appropriate credit to the original author(s) and the source, provide a link to the Creative Commons license, and indicate if changes were made. 
performance are the radionuclide $\left({ }^{82} \mathrm{Rb}\right)$ kinetic properties, better resolution and sensitivity PET scanners, and the accurate attenuation correction (AC) achievable in modern PET/CT imaging [6-8]. Furthermore, the use of cardiac ${ }^{82} \mathrm{Rb} \mathrm{PET} / \mathrm{CT}$ has been promoted by its ability to quantify myocardial blood flow (MBF) and myocardial flow reserve (MFR) noninvasively and routinely $[9,10]$. Accuracy and precision in quantitative MBF ${ }^{82} \mathrm{Rb}$ dynamic PET imaging have been recently reviewed [11] while reproducible results can be achieved with the available software [12].Improvement of PET quantitative cardiac imaging can be obtained by optimization of acquisition parameters [1315]. Present generation PET/CT includes time-of-flight (TOF) information to better recover the localization of positron annihilations sites [13], with consequent statistical noise reduction and increase in lesion contrast [16-18] and benefits in ${ }^{82} \mathrm{Rb}$ PET perfusion studies of obese patients [19]. Furthermore, this method seems less sensitive to mismatched attenuation correction, erroneous normalization, and poorly estimated scatter correction, but such robustness also depends on the time resolution of the TOF-PET scanner [16-18, 20, 21]. Recently, tomographic reconstruction algorithms including point spread function (PSF) recovery became available. PSF inclusion in the iterative tomographic reconstruction process leads to valuable improvements of spatial resolution with consequent reduction of partial volume effects (PVE) translating in increased activity recovery and lesion contrast [22]. Because spurious events may also appear erroneously in regions of the image where there is no true activity, the implementation of robust scatter and attenuation corrections appears essential [17, 23-25]. On the other hand, the quantification of MBF and MFR in ${ }^{82} \mathrm{Rb}$ PET images might also be affected by reconstruction type (iterative or analytical). Cardiac PET image reconstruction can be achieved by both filtered back projection (FBP) and iterative reconstruction algorithms [26]. The filtered back projection (FBP) method is fast, linear, and robust and often still is the preferred reconstruction method for dynamic PET studies consisting of many frames with short scan durations and thus poor statistics [27-29]. However, reconstructed images by FBP may contain severe streak artifacts, increasing image noise and reducing contrast, which may mask lesions [27-29]. Thus, FBP has been replaced by iterative algorithms in oncological PET, as their reconstruction times became clinically acceptable. Consequently, the principal method used is the iterative ordered subset expectation maximization (OSEM), which processes the projection data subset by subset instead of all the data at once [27]. The aim of this study was to investigate the quantitative influence of TOF and PSF recovery corrections, as well as reconstruction parameters (subsets $\times$ iteration product; filters) used in OSEM algorithms for cardiac ${ }^{82} \mathrm{Rb} \mathrm{PET} / \mathrm{CT}$, as a first step towards optimization or recommendations.

\section{Methods}

\section{Study population}

Twenty adult patients were referred for cardiac ${ }^{82} \mathrm{Rb}$ PET/CT and underwent rest and adenosine stress studies at the Lausanne University Hospital. The data included in this study were retrospectively collected between September and December 2013. Patients were divided into two groups: $G_{1}(n=10$ patients without myocardial flow defects) and $\mathrm{G}_{2}(n=10$ patients with myocardial flow abnormalities, such as sequels of myocardial infarction $(n=2 ; 20 \%)$, stress ischemia $(n=3$; 
$30 \%$, SDS $\geq 3)$, or globally diminished myocardial flow reserve $(n=5 ; 50 \%))$. Patient characteristics are described in Table 1.

\section{Patient preparation}

Written informed consent was obtained for all patients before inclusion, and history taking covering symptoms, risk factors, diseases, medication, and prior diagnostic or therapeutic procedures was performed. Patients were refrained from taking caffeinecontaining substances for at least $24 \mathrm{~h}$ and were asked to fast at least $6 \mathrm{~h}$ prior to the PET studies; medications that may interfere with the cardiac pharmacological stress agent (e.g., nitrates or beta-blockers) were suspended for $\geq 24 \mathrm{~h}$.

\section{Protocol and image acquisition}

All dynamic studies were performed using a 3D PET/CT scanner (Discovery 690; GE Healthcare, Waukesha, WI) equipped with cerium-doped lutetium yttrium orthosilicate (LYSO) scintillation crystals (13824 LYSO crystals of size: $4.2 \times 6.3 \times 25 \mathrm{~mm} 2$ ); axial field of view (FOV) is $157 \mathrm{~mm}$ while transaxial FOV is $70 \mathrm{~cm}$. Annihilation photons were acquired in a $425-650 \mathrm{keV}$ energy window. TOF coincidence-timing resolution is $550 \mathrm{ps}$. Emission images at rest were obtained after ${ }^{82} \mathrm{RbCl}$ administration of $10 \mathrm{MBq} /$ $\mathrm{kg}$ (max $1300 \mathrm{MBq}$ ) by an automated system $\left({ }^{82} \mathrm{Sr} /{ }^{82} \mathrm{Rb}\right.$ generator). Pharmacological stress was induced with adenosine $(140 \mu \mathrm{g} / \mathrm{kg} / \mathrm{min})$ to obtain stress perfusion results. A second injection of radiopharmaceutical $(10 \mathrm{MBq} / \mathrm{kg})$ was injected $\geq 10.1 \mathrm{~min}$ after the first injection, at the end of the second minute of adenosine intravenous infusion. A list-mode acquisition was started simultaneously with radiopharmaceutical injection for both studies (rest, stress), and dynamic images were reconstructed (21 frames, 35 slices each; $12 \times 8,5 \times 12,1 \times 30,1 \times 60,1 \times 120$, and $1 \times 240 \mathrm{~s}$ ) for deriving absolute flow measurement. The attenuation maps for rest and stress studies were obtained from the imbedded 64-slice CT scanner adopting a low-dose CT setup $(120 \mathrm{kV}, 10 \mathrm{~mA}, 0.8 \mathrm{~s}$ per

Table 1 Patient characteristics according to the group without and with myocardial flow defects (mean \pm SD or $n(\%)$ ) according to standard reconstruction protocol (protocol A in Table 2)

\begin{tabular}{lll}
\hline & $\begin{array}{l}\text { Patients without myocardial } \\
\text { flow defects }(n=10)\end{array}$ & $\begin{array}{l}\text { Patients with myocardial } \\
\text { flow defects }(n=10)\end{array}$ \\
\hline Age (years) & $62 \pm 9$ & $68 \pm 9$ \\
Sex (female/male) & $3 / 7(30 \% / 70 \%)$ & $2 / 8(20 \% / 80 \%)$ \\
BMI (kg/m²) & $27 \pm 6$ & $29 \pm 7$ \\
Hypertension & $5(50 \%)$ & $5(50 \%)$ \\
Diabetes mellitus & $2(20 \%)$ & $3(30 \%)$ \\
Dyslipidemia & $3(30 \%)$ & $3(30 \%)$ \\
Current smokers & $2(20 \%)$ & $0(0 \%)$ \\
Prior myocardial infarction & $0(0 \%)$ & $2(20 \%)$ \\
Ischemia & $0(0 \%)$ & $3(30 \%)$ \\
Decreased MFR & $0(0 \%)$ & $5(50 \%)$ \\
LV rest MBF (mL/min/g) & $1.10 \pm 0.31$ & $0.84 \pm 0.24$ \\
LV stress MBF (mL/min/g) & $3.08 \pm 0.58$ & $1.50 \pm 0.63$ \\
LV MFR [1] & $3.03 \pm 1.22$ & $1.76 \pm 0.51$ \\
\hline BMI body mass index, LV left vis
\end{tabular}

$B M I$ body mass index, $L V$ left ventricle, MBF myocardial blood flow, MFR myocardial flow reserve 
rotation, pitch 1.375:1). The first CT was acquired after the scout scan for accurate definition of the axial examination range and just before the rest study; the second CT scan was repeated immediately after the stress acquisition. All stress procedures were supervised by a qualified physician with knowledge of pharmacological stress agents and expertise in advanced life support techniques.

\section{OSEM reconstruction protocols}

Reconstruction protocols (RP) with Gaussian filters of 4, 6, and $8 \mathrm{~mm}$ and variable iterations and subsets (iterations $\times$ subsets) of $2 \times 24,2 \times 16,3 \times 16$, and $4 \times 16$ were created, as described in the Table 2. A total of 320 reconstructions were performed, 20 for each patient (including rest and stress dynamic studies). The standard reference RP used in the nuclear medicine department for dynamic studies with ${ }^{82} \mathrm{Rb}$ was OSEM + TOF with OSEM reconstruction 2 iterations $\times 24$ subsets and a full-width half maximum (FWHM) filter of $6 \mathrm{~mm}$. In the absence of a quantitative "gold standard," to investigate the influence of reconstruction parameters (TOF, PSF, iterations $\times$ subsets, and filters) in the quantification of MBF and MFR, we compared results obtained from the tested RPs with our standard reference protocol.

Image reconstruction and processing

About $14 \%$ of ${ }^{82} \mathrm{Rb} \beta+$ decays also produce a $776.5 \mathrm{keV} \gamma$-ray with no angular correlation. This results in a significant pollution of the true coincidence emission data, distinct in shape from the scatter component, and must be corrected in view of quantitative cardiac perfusion imaging [27, 30, 31]. Quantitative PET reconstructions were corrected for prompt gamma pollution using a specific correction method provided by the vendor (not yet reported in the literature). Image quality control (QC) was used to obtain a precise alignment of the heart in PET and CT images in the short axis, vertical, and horizontal long-axis views. Rest and stress dynamic studies were reconstructed from list mode.

\section{Quantitative analysis of MBF and MFR}

${ }^{82} \mathrm{Rb}$ time activity curves (TACs) were used to generate automatically segmental, regional, and global MBF, MFR (stress/rest), and myocardial flow difference (stress-rest) polar maps using FlowQuant $\odot$ software (Ottawa Heart Institute, Ottawa, CA) [32, 33].

Table 2 Reconstruction protocols (changes to standard protocol are marked in italics)

\begin{tabular}{llll}
\hline Reconstruction protocols & Iterations $\times$ subsets & Filter FWHM $(\mathrm{mm})$ & Difference vs. standard protocol (A) \\
\hline (A) OSEM + TOF & $2 \times 24=48$ & 6 & - \\
(B) OSEM + TOF & $4 \times 16=64$ & 6 & Higher iterations $\times$ subsets product \\
(C) OSEM + TOF & $3 \times 16=48$ & 4 & Lower smoothing \\
(D) OSEM + TOF & $2 \times 16=32$ & 6 & Lower iterations $\times$ subsets product \\
(E) OSEM + TOF + PSF & $3 \times 16=48$ & 6 & With PSF recovery \\
(F) OSEM + TOF & $3 \times 16=48$ & 8 & Higher smoothing \\
(G) OSEM + TOF & $3 \times 16=48$ & 6 & Same product of iterations $\times$ subsets, \\
(H) OSEM + no TOF & $3 \times 16=48$ & 6 & Do TOF correction \\
\hline
\end{tabular}

OSEM ordered subsets expectation maximization, TOF time of flight, PSF point spread function 
According to the American Heart Association (AHA) recommendations for the left ventricle (LV) segmentation [34], each polar map (rest, stress) was divided into 17 different segments corresponding to the LV myocardium, which can be assigned to one of the three coronary artery territories. A 1-tissue compartment modeling procedure was used to calculate MBF and MFR in absolute terms $(\mathrm{mL} / \mathrm{min} / \mathrm{g})$ [31-33]. This model included an input function derived from arterial blood in the base of the left ventricle, regional uptake and clearance parameters $\left(K_{1}, \mathrm{~mL} / \mathrm{min} / \mathrm{g}\right.$ and $\left.k_{2}, \mathrm{~min}^{-1}\right)$, blood to myocardium spillover fraction $f_{b}$, and myocardial partial volume correction $\left(1-f_{b}\right)$. The

${ }^{82} \mathrm{Rb}$ extraction fraction was used to estimate MBF and consequently MFR (stress/rest) from $K_{1}$. The activity concentration in the myocardium $C_{\mathrm{m}}(t)$ was calculated by:

$$
C_{m}(t)=K_{1} \cdot e^{-k 2 \cdot 1} \otimes C_{\mathrm{LV}}(t),
$$

where $K_{1}$ and $k_{2}$ represent the uptake and washout constants and $C_{\mathrm{LV}}(t)$ the activity concentration of the blood in the $\mathrm{LV}$, and $\otimes$ represents the convolution operator.

${ }^{82} \mathrm{RbCl}$ does not accumulate in/or clear from myocardium proportionally linear to the perfusion caused by an incomplete and curvilinear myocardial extraction fraction from arterial blood with increasing flow rates [8]. Parametric images representing a graphical illustration of quantitative MBF were then generated to display perfusion at the voxel level, based on the evaluation of the tracer kinetic model for each voxel [33]. Voxels were also grouped to give MBF according to each of the 17 AHA segment and then according to coronary artery territories and finally the whole LV [34].

\section{Statistical methods}

The differences to the standard clinical reconstruction protocol were analyzed using Lin's concordance correlation coefficient and the Bland-Altman (BA) plots [35, 36]. In Lin's concordance coefficient $\rho_{\mathrm{c}}$ is considered as a measure of precision and accuracy to assess the degree of agreement between two measures of the same variables performed with different reconstruction parameters [35]. In all cases, a perfect concordance gives $\rho_{\mathrm{c}}=1$ and the absence of concordance $\rho_{\mathrm{c}}=0$. This approach offers several advantages to compare measurements of the same value and has been used to compare MBF measurements obtained from several software packages [32]. Box plots with median and quartile (upper and lower) values were created for rest, stress, and reserve results of all RP. For BA analysis, we calculated the mean ( $x$-axis) and the differences ( $y$-axis) between each RP comparison to obtain a graph to evaluate the discrepancy of values in the rest and stress dynamic studies.

The 95\% confidence interval limits of concordance were included in all graphs and tables. Using these limits, it is easily seen if two concordance coefficients are alike or different at the $p<0.05$ level just by checking for the presence of overlapping $95 \% \mathrm{CI}$ limits (no difference between methods as concordance coefficients or $p>0.05$ ) or not (significant difference between methods with $p \leq 0.05$ ).

All statistical analyses were performed with Stata 13.0 software (Stata Corporation, College Station, TX) using a $p$ value $<0.05$ as the significance level.

\section{Results}

Lin's concordance coefficients for rest, stress MBF, and MFR in the three coronary artery territories and 17 segments, as shown in Tables 3 and 4, respectively, as well as in 
Table 3 Lin's concordance coefficient $\rho_{c}$ obtained for MBF and MFR comparisons in the three coronary territories (LAD, LCX, RCA)

\begin{tabular}{llll}
\hline RP comparisons & \multicolumn{3}{l}{ All patients $(n=60$ coronary territories) } \\
\cline { 2 - 4 } & $\rho_{\mathrm{c} \text { (Rest) }}[95 \% \mathrm{Cl}]$ & $\rho_{\mathrm{c}(\text { Stress) }}[95 \% \mathrm{Cl}]$ & $\rho_{\mathrm{c}(\mathrm{MFR})}[95 \% \mathrm{Cl}]$ \\
\hline B vs. A & $0.955[0.933 ; 0.977]$ & $0.983[0.975 ; 0.992]$ & $0.939[0.910 ; 0.967]$ \\
C vs. A & $0.966[0.949 ; 0.982]$ & $0.984[0.976 ; 0.992]$ & $0.909[0.847 ; 0.972]$ \\
D vs. A & $0.939[0.836 ; 0.959]$ & $0.985[0.977 ; 0.992]$ & $0.957[0.928 ; 0.987]$ \\
E vs. A & $0.951[0.927 ; 0.976]$ & $0.990[0.985 ; 0.995]$ & $0.883[0.808 ; 0.958]$ \\
F vs. A & $0.968[0.954 ; 0.982]$ & $0.984[0.976 ; 0.992]$ & $0.898[0.836 ; 0.960]$ \\
G vs. A & $0.938[0.909 ; 0.967]$ & $0.986[0.980 ; 0.992]$ & $0.876[0.807 ; 0.845]$ \\
H vs. A & $0.769^{*}[0.678 ; 0.861]$ & $0.789^{*}[0.789 ; 0.912]$ & $0.880[0.805 ; 0.955]$ \\
\hline
\end{tabular}

${ }^{*} p<0.05$ vs. other comparisons (in italics in same column)

Tables 5 and 6, according to the presence or absence of perfusion abnormalities (Figure 1). The difference in values between each RP and standard setting was used to identify which changes to the reconstruction parameters affected the MBF or MFR the most, based on the overlapping or not of the $95 \%$ CI given in the tables, defining whether any comparison was significant at the $p<0.05$ value level.

The results of comparisons between the different combination of RP and the standard protocol used (RP A: OSEM + TOF; 2 iterations $\times 24$ subsets, FWHM $=6 \mathrm{~mm}$ ) showed a good correlation in the majority of cases, with concordance values close to $\rho_{\mathrm{c}}=1.0$. However, the concordance was significantly lower for the RP H without TOF in the rest and stress MBF for patient groups $G_{1}$ and $G_{2}$ in the coronary artery territories $(p<0.05$, Table 5$)$ and in the 17 -segment evaluation $(p<0.05$, Table 6$)$. The statistical analyses applied to MFR results for $G_{2}$ also reveal significant differences for the same RP without TOF (protocol $\mathrm{H}$ ). On the other hand, MFR values for $\mathrm{G}_{1}$ exhibited no significant difference compared to the reference standard RP on a coronary territory level (Table 5), while it was significant on the 17-segment level (Table 6). BA plots (Figure 2) depict the differences in rest and stress data reconstructed by protocols A and $\mathrm{H}$. The largest changes could be observed once more in reconstruction without TOF (Figure 3). Similar differences existed when comparing protocol G vs. protocol H, which only differed by the removal of TOF (data not shown). In contrast, both rest and stress studies reconstructed with OSEM + TOF + PSF showed less dispersion when compared to the standard protocol (Figure 4). Changes in iteration and subset numbers

Table 4 Lin's concordance coefficient $\rho_{c}$ obtained for MBF and MFR comparisons in the 17segment model

\begin{tabular}{llll}
\hline RP comparisons & \multicolumn{3}{l}{ All patients $(n=340$ segments) } \\
\cline { 2 - 4 } & $\rho_{\text {c (Rest) }}[95 \%$ Cl] & $\rho_{\text {c (Stress) }}[95 \%$ Cl $]$ & $\rho_{\text {c (MFR) }}[95 \%$ Cl] \\
\hline B vs. A & $0.954[0.945 ; 0.964]$ & $0.982[0.978 ; 0.986]$ & $0.938[0.926 ; 0.950]$ \\
C vs. A & $0.966[0.959 ; 0.973]$ & $0.982[0.978 ; 0.986]$ & $0.945[0.933 ; 0.956]$ \\
D vs. A & $0.943[0.933 ; 0.954]$ & $0.982[0.978 ; 0.986]$ & $0.971[0.965 ; 0.977]$ \\
E vs. A & $0.954[0.944 ; 0.964]$ & $0.988[0.985 ; 0.990]$ & $0.931[0.918 ; 0.944]$ \\
F vs. A & $0.962[0.955 ; 0.970]$ & $0.980[0.976 ; 0.984]$ & $0.941[0.930 ; 0.952]$ \\
G vs. A & $0.944[0.933 ; 0.955]$ & $0.983[0.980 ; 0.987]$ & $0.926[0.912 ; 0.939]$ \\
H vs. A & $0.794^{*}[0.759 ; 0.829]$ & $0.857^{*}[0.833 ; 0.882]$ & $0.893^{*}[0.872 ; 0.914]$ \\
\hline
\end{tabular}

${ }^{*} p<0.05$ vs. other comparisons (in italics in same column) 


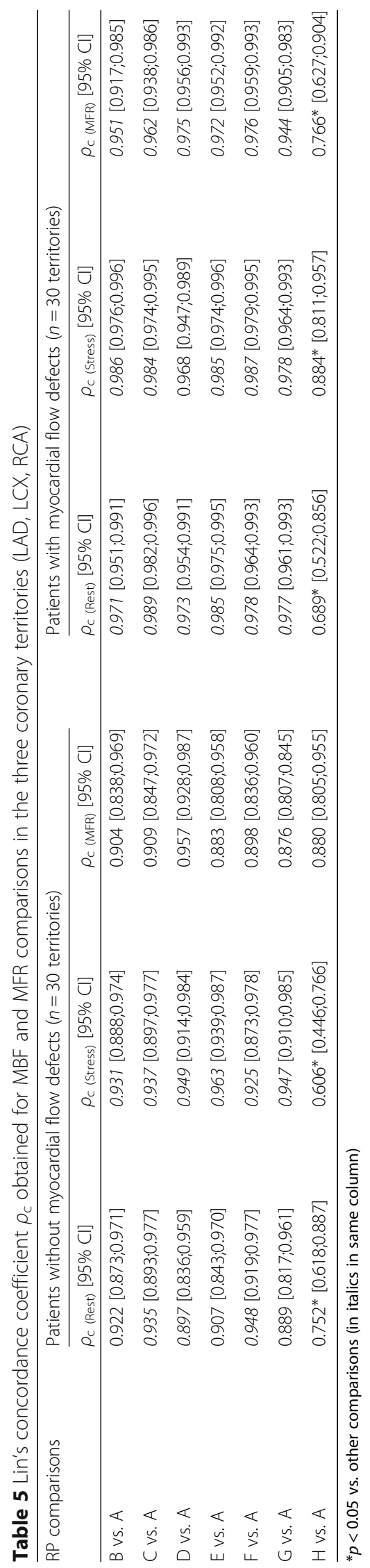




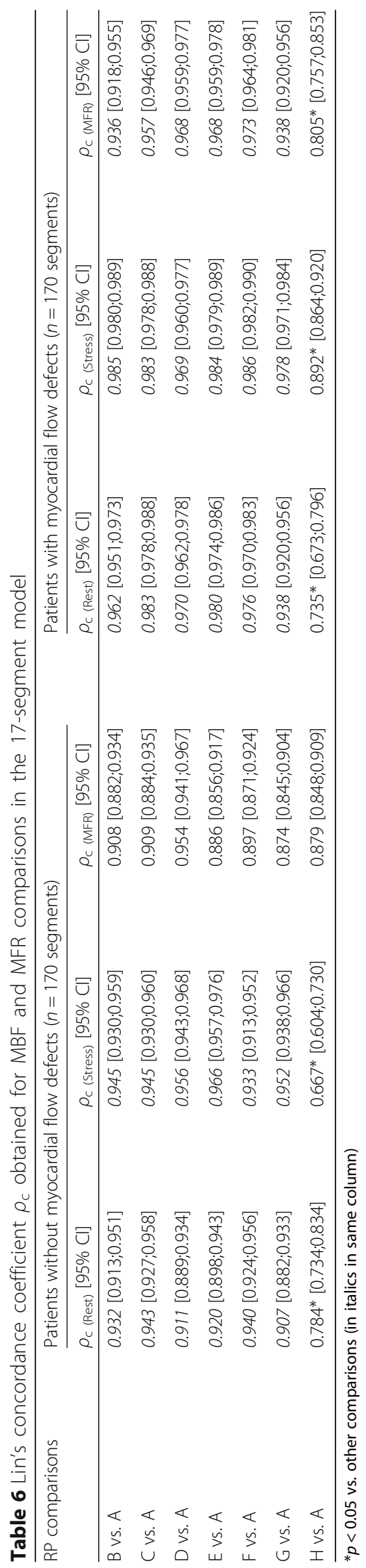



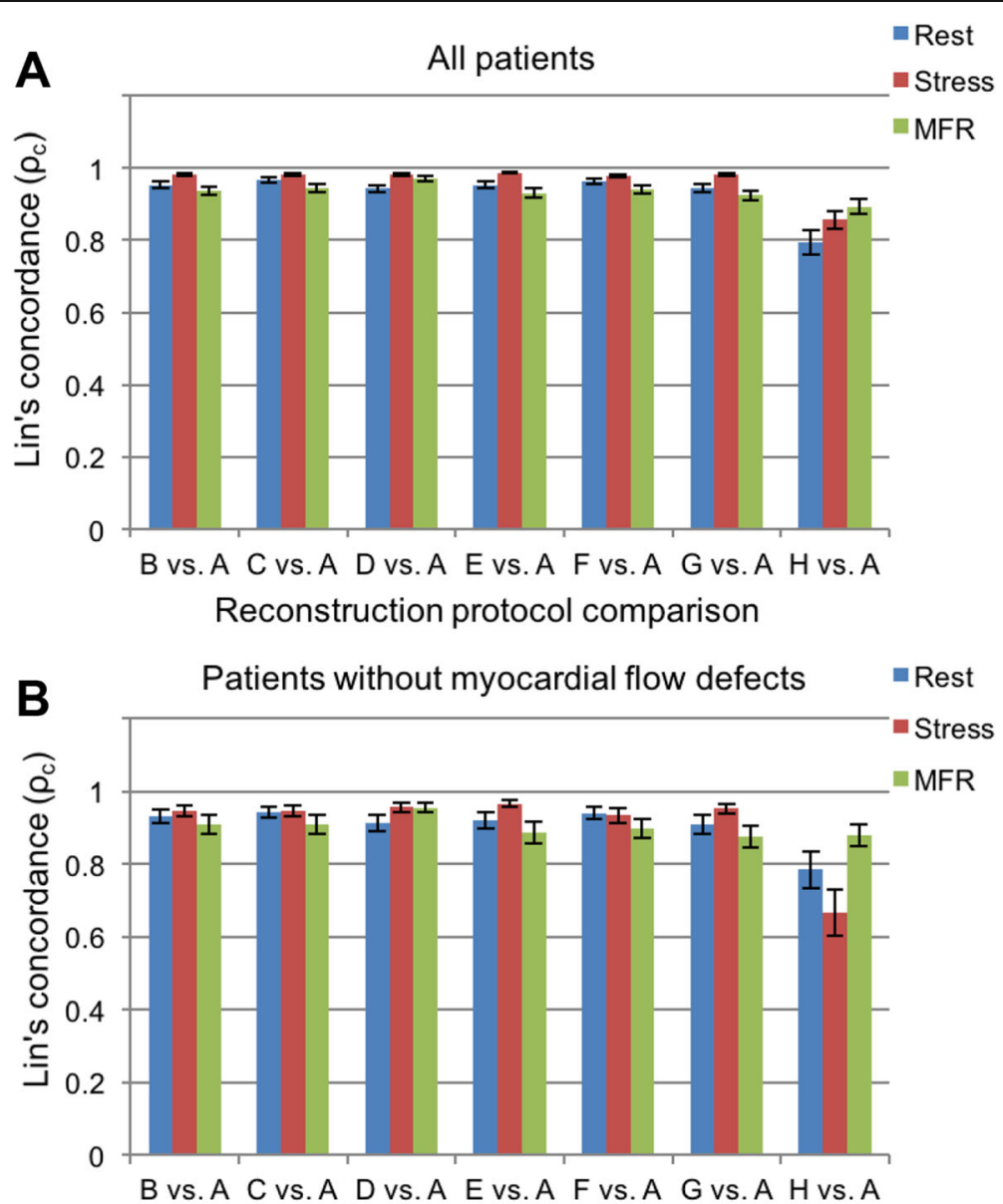
Reconstruction protocol comparison

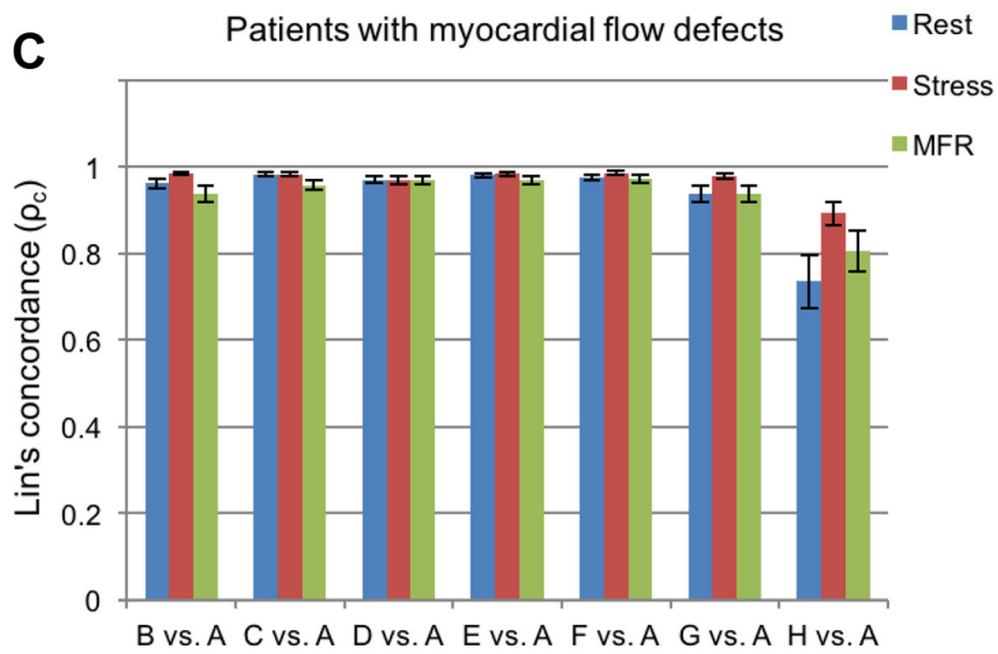

Reconstruction protocol comparison

Fig. 1 Concordance coefficients $\rho_{c}$ for rest, stress MBF, and myocardial flow reserve (MFR) for: a all patients, b patients without myocardial flow defects, and $\mathbf{c}$ patients with myocardial flow defects in segmental analysis 


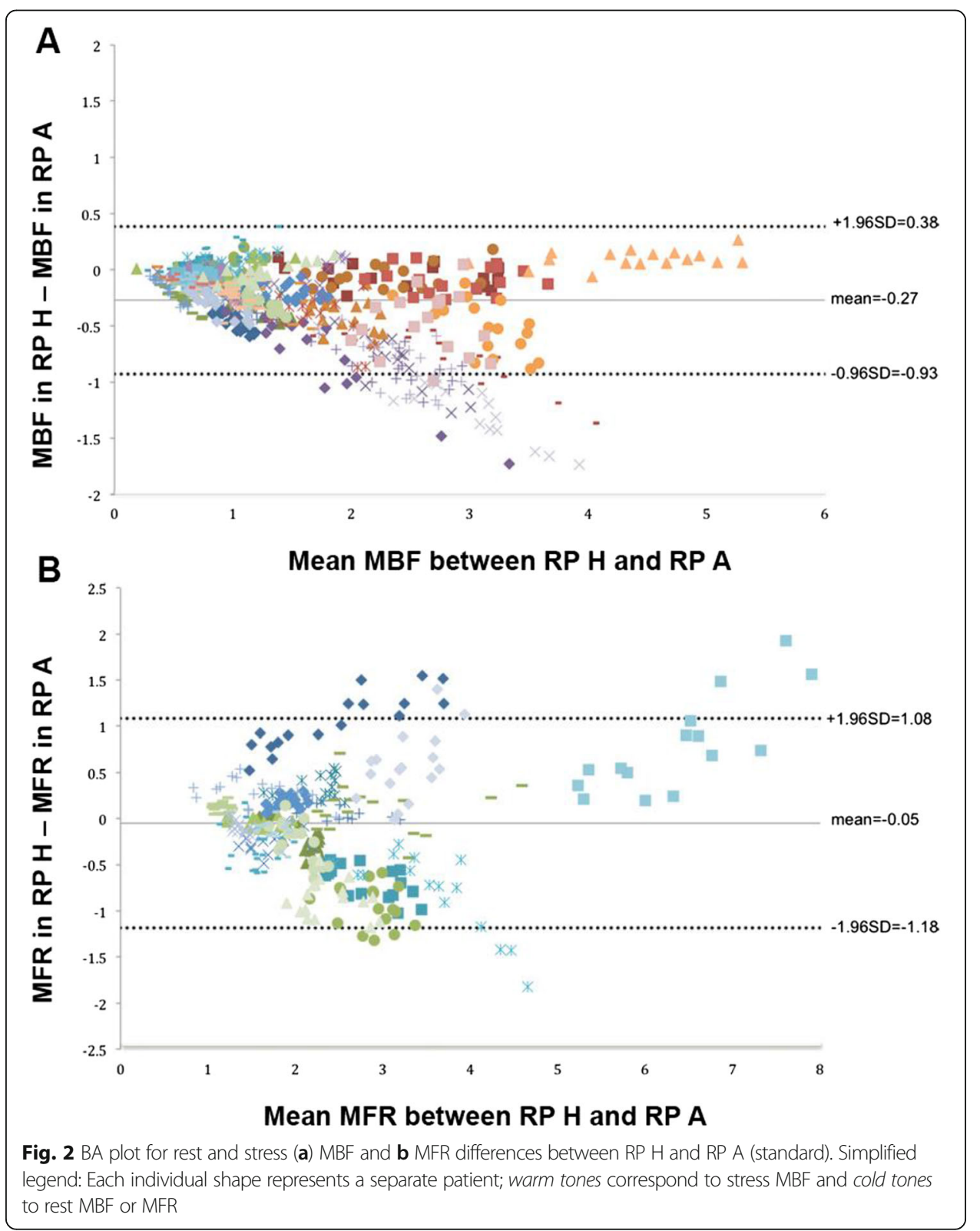

showed to have minimal influence on MBF in contrary to removal of TOF. In individual segments, these changes could reach up to $1.7 \mathrm{~mL} / \mathrm{min} / \mathrm{g}$.

\section{Discussion}

Our investigations were performed to evaluate the effect of reconstruction parameters and different algorithms in quantitative cardiac ${ }^{82} \mathrm{Rb} \mathrm{PET} / \mathrm{CT}$ imaging. Over the years, changes in reconstruction parameters have been made mainly for ${ }^{18} \mathrm{~F}-\mathrm{FDG}$ PET/CT and can be considered crucial for improving image quality. However, few studies have been specifically performed in cardiac PET imaging to study the influence of reconstruction parameters on image reconstruction quality in clinically relevant condition with ${ }^{82} \mathrm{Rb}$. 


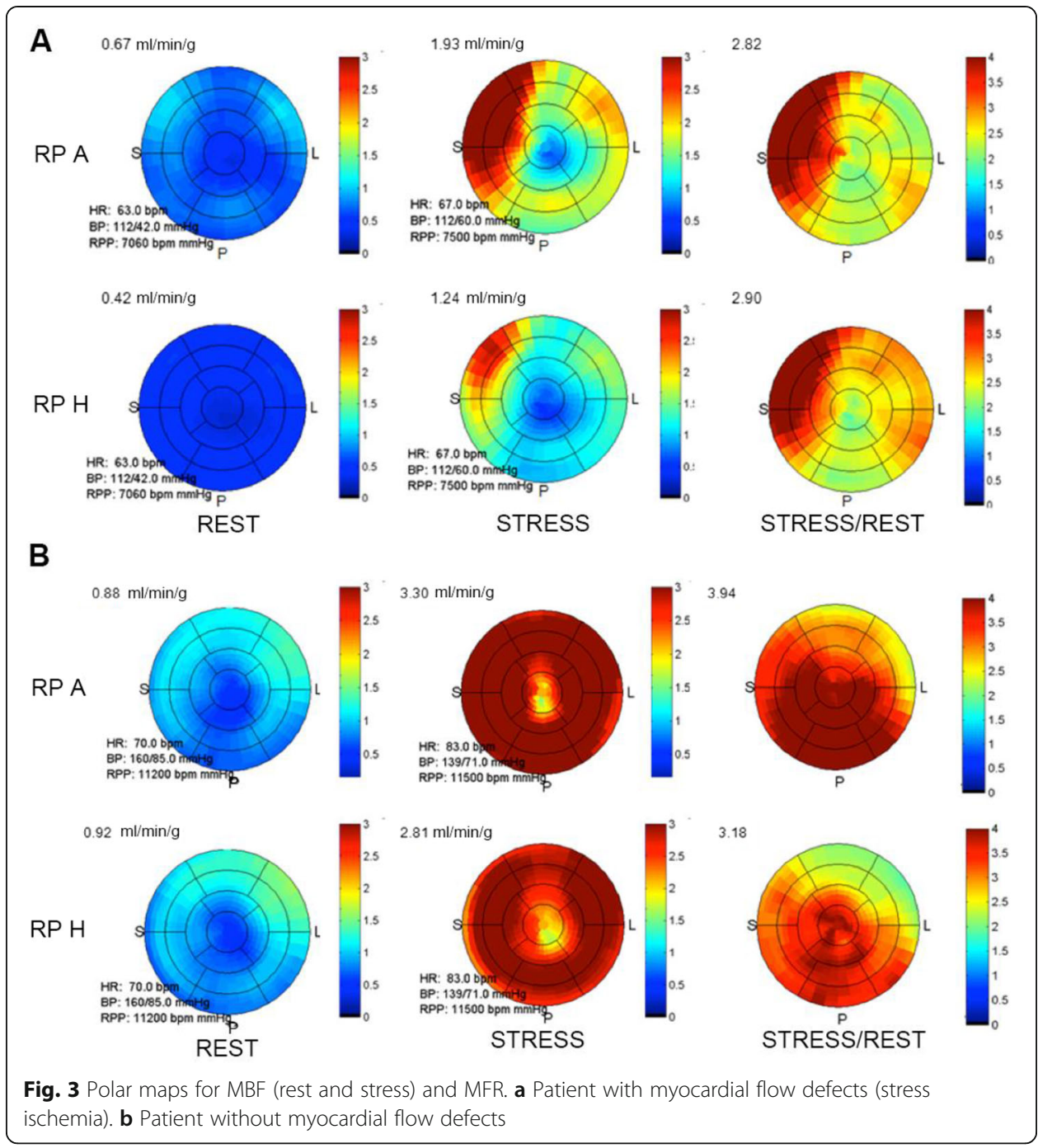

The benefit arising from the combined use of TOF and PSF corrections in quantitative cardiac PET has been investigated by Presotto et al. [37]; this study was limited to only a phantom setup filled with ${ }^{18} \mathrm{~F}$-FDG. Because of the short half-life of ${ }^{82} \mathrm{Rb}$ and the relative high activity to handle, realistic phantom studies in clinical relevant condition are not easy to perform. Nevertheless, recently, Renaud at al. [38] tested the MBF quantitative accuracy in ${ }^{82} \mathrm{Rb}$ cardiac PET in different PET scanners. In particular, the specific activity administered to patients in our study $(10 \mathrm{MBq} / \mathrm{kg})$ is very close to the optimal value (11.4 MBq/kg) found by Renaud et al. for the employed PET scanner (GE Discovery 690). Thus, we do not expect quantitative bias due to the total activity of radiotracer administered to patients, especially at the fist pass bolus transit.

In our study, different RPs were created and compared with a reference standard protocol used routinely in our nuclear medicine department, to understand the impact of TOF and PSF corrections included in OSEM reconstruction algorithms. The influence of the number of iterations, subsets, and Gaussian filtering on MBF and MFR quantification was also assessed. The number of subsets used in RP was 16 or 24 to avoid image degradation (i.e., noise, artifacts), as PET image quality 


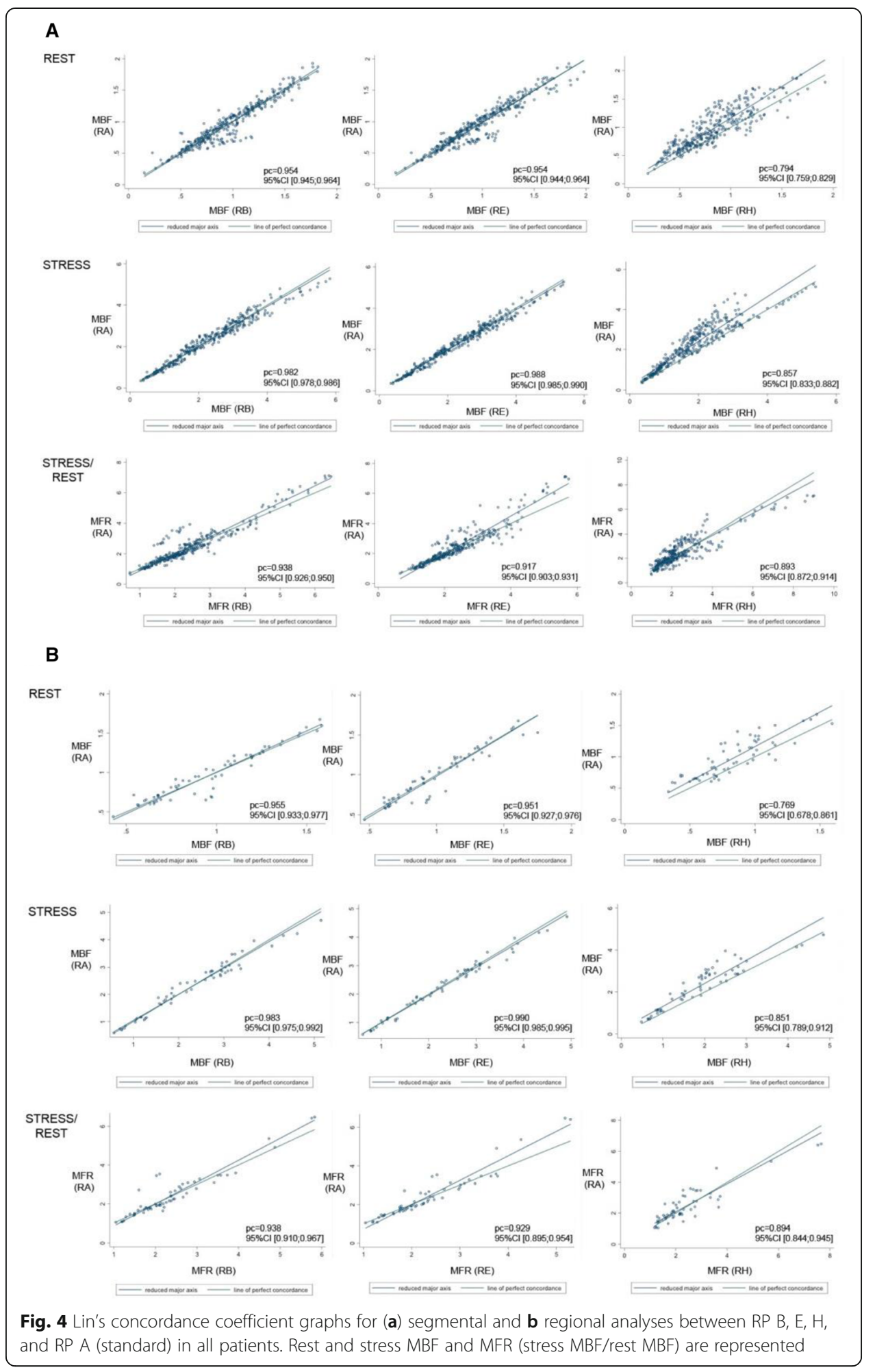

with OSEM declined as the number of subsets increased, even when the TOF algorithm was used [39].

Rest and stress dynamic studies reconstructed without TOF showed a significant difference in global and regional MBF values, comparatively to the standard protocol, in 
$\mathrm{G}_{1}$ and $\mathrm{G}_{2}$. This can be explained because TOF algorithm is crucial to improve image quality and lesion contrast potentially providing more reliable results. The largest differences in MBF values, in relation to the reference standard protocol, appeared in the RP without TOF correction. Maebatake et al. used phantom studies based on the relationship between the noise equivalent count (NEC), as an index of PET image quality and TOF-PET image quality for ${ }^{18}$ F-FDG PET/CT showing similar results regarding the impact of PSF and TOF [40]. Significant improvements on image contrast were observed in the OSEM reconstructions combined with the PSF algorithm. The study also highlighted that similar coefficients of variance are obtained for nonTOF and TOF reconstructions; however, a longer acquisition time is required for those without TOF to obtain identical image contrast and reduce levels of noise [40]. For the TOF + PSF RPs, no major differences were revealed from the standard, presenting both high $\rho_{\mathrm{c}}$ values, which means a good correlation between two study variables. According to the results of Akamatsu et al. [41], the combination TOF + PSF in ${ }^{18}$ F-FDG PET/CT clearly improves image quality, showing a more uniform background. In our study, we did not observe a significant difference by adding PSF recovery; we can hypothesize that this might not have a big effect as the heart is moving and thus blurring the image anyway. A few patients had small hearts, which can increase the spillover effect (due to the proximity of blood pool volume of interest and myocardium) and underestimate MBF values obtained for both groups of patients in OSEM reconstruction.

The present study had some limitations. First, the number of patients included in this study could have been bigger in order to improve the statistical pertinence of the presented results. Second, a phantom study providing gold standard values for MBF and MFR (normal database) would have been beneficial but very difficult to perform with such a so short-lived radiotracer. Third, there might have been the presence of patient with high stress flows and reserve, who might have biased our results, but as this was real patient data, we decided not to exclude them as they are observed in clinical practice. Fourth, it is also difficult to evaluate whether the observed differences have a true clinical significance or not. As pinpointed by Moody et al., statistical uncertainty in the Renkin-Crone relation account for $50-70 \%$ of the coefficient of variation at rest and $40-50 \%$ at stress [11], so that we can only evaluate if the different parameters produce similar or discrepant results. Fifth, the comparison of concordance coefficient between $G_{1}$ and $G_{2}$ might have been influenced by the dynamic range of MBF and MFR.

Furthermore, a RP with OSEM + PSF without TOF would be interesting to study for understanding the role of PSF correction alone in the quantification of MBF and MFR.

Finally, additional prospective investigations with FBP algorithm are warranted to explore the main differences between analytical and iterative reconstructions in ${ }^{82} \mathrm{Rb}$ dynamic PET/CT measurements.

\section{Conclusions}

Changes in reconstruction parameters applied to rest and stress ${ }^{82} \mathrm{Rb}$ perfusion studies influenced MBF and MFR quantitation. Concordance among the different reconstruction protocols was excellent and most variations had little effect, except for the exclusion of TOF, which significantly reduced concordance of rest and stress MBF, as well as MFR, both at the coronary territory and segmental level. 


\begin{abstract}
Abbreviations
${ }^{82} \mathrm{Rb}$ : Rubidium-82; ${ }^{82} \mathrm{Sr} /{ }^{82} \mathrm{Rb}$ : Strontium-82/Rubidium-82; AC: Attenuation correction; BA: Bland-Altman; CAD: Coronary arterial disease; FBP: Filtered back projection; FOV: Field of view; FWHM: Full-width half maximum; LV: Left ventricle; LYSO: Lutetium yttrium orthosilicate; MBF: Myocardial blood flow; MFR: Myocardial blood reserve; NEC: Noise-equivalent count; OSEM: Ordered subset expectation maximization; PET/CT: Positron emission tomography/computed tomography; Prompt: Gamma; PSF: Point spread function; PVE: Partial volume effects; QC: Quality control; RP: Reconstruction protocol; SPECT: Single photon emission computerized; TACs: Time activity curves; TOF: Time of flight
\end{abstract}

\title{
Acknowledgements
}

We acknowledge the grant of the European Union Mobility Program Leonardo Da Vinci awarded to the first author (P. Chilra).

\section{Authors' contributions}

PC carried out the data acquisition analysis and interpretation of the data, drafted the manuscript first version. SG, GA, carried out the data analysis and interpretation of the data, drafted the manuscript. MSM carried out the interpretation of the data, helped draft the manuscript revising it critically. JOP conceived the study, participated in its design and coordination, and revised the manuscript critically as well as the resubmitted manuscript. LV participated to the conceived of the study and revised the manuscript critically. JAPJ participated to the conceived of the study, participated in its design and coordination, and revised the manuscript critically. All authors gave the final approval of the version to be published.

\section{Competing interests}

The authors declare that they have no competing interests.

\section{Ethics approval and consent to participate}

The research protocol was authorized by the cantonal (Canton of Vaud, Switzerland) ethics committee. Informed consent was obtained from all individual participants included in the study.

\section{Author details}

${ }^{1}$ Haute École de Santé Vaud - Filière TRM, University of Applied Sciences and Arts Western Switzerland, Lausanne, Switzerland. ${ }^{2}$ Institute of Radiation Physics, Lausanne University Hospital, Lausanne, Switzerland. ${ }^{3}$ Nuclear Medicine and Molecular Imaging Department, Lausanne University Hospital, Lausanne, Switzerland. ${ }^{4}$ Área Científica de Medicina Nuclear, Escola Superior de Tecnologia da Saúde de Lisboa, Instituto Politécnico de Lisboa, Lisbon, Portugal. ${ }^{5}$ Instituto de Biofísica e Engenharia Biomédica, Faculdade de Ciências da Universidade de Lisboa, Lisbon, Portugal.

\section{Received: 16 December 2015 Accepted: 8 February 2017}

Published online: 15 February 2017

\section{References}

1. Flotats A, Knuuti J, Gutberlet M, Marcassa C, Bengel FM, Kaufmann PA, et al. Hybrid cardiac imaging: SPECT/CT and PET/CT. A joint position statement by the European Association of Nuclear Medicine (EANM), the European Society of Cardiac Radiology (ESCR) and the European Council of Nuclear Cardiology (ECNC). Eur I Nucl Med Mol Imaging. 2011;38(1):201-12.

2. Dilsizian V. Highlights from the updated joint ASNC/SNMMI PET myocardial perfusion and metabolism clinical imaging guidelines. J Nucl Med. 2016;57(9):1327-8.

3. Tilkemeier PL, Cooke CD, Grossman GB, McCallister BD, Ward RP. Standardized reporting of radionuclide myocardial perfusion and function. J Nucl Cardiol. 2009;16(4):650-.

4. Alvarez-Diez TM, deKemp R, Beanlands R, Vincent J. Manufacture of strontium-82/rubidium-82 generators and quality control of rubidium- 82 chloride for myocardial perfusion imaging in patients using positron emission tomography. Appl Radiat Isot. 1999;50(6):1015-23.

5. Bateman TM, Heller GV, McGhie Al, Friedman JD, Case JA, Bryngelson JR, et al. Diagnostic accuracy of rest/stress ECG-gated Rb-82 myocardial perfusion PET: comparison with ECG-gated Tc-99m sestamibi SPECT. J Nucl Cardiol. 2006;13(1):24-33.

6. Mc Ardle BA, Dowsley TF, deKemp RA, Wells GA, Beanlands RS. Does rubidium-82 PET have superior accuracy to SPECT perfusion imaging for the diagnosis of obstructive coronary disease?: A systematic review and meta-analysis. J Am Coll Cardiol. 2012;60(18):1828-37.

7. Santana CA, Folks RD, Garcia EV, Verdes L, Sanyal R, Hainer J, et al. Quantitative (82)Rb PET/CT: development and validation of myocardial perfusion database. J Nucl Med. 2007;48(7):1122-8.

8. Lortie M, Beanlands RS, Yoshinaga K, Klein R, Dasilva JN, DeKemp RA. Quantification of myocardial blood flow with 82Rb dynamic PET imaging. Eur J Nucl Med Mol Imaging. 2007;34(11):1765-74.

9. Prior JO, Allenbach G, Valenta I, Kosinski M, Burger C, Verdun FR, et al. Quantification of myocardial blood flow with 82Rb positron emission tomography: clinical validation with 150-water. Eur J Nucl Med Mol Imaging. 2012; 39(6):1037-47.

10. Ziadi MC, Dekemp RA, Williams K, Guo A, Renaud JM, Chow BJ, et al. Does quantification of myocardial flow reserve using rubidium- 82 positron emission tomography facilitate detection of multivessel coronary artery disease? J Nucl Cardiol. 2012;19(4):670-80.

11. Moody JB, Murthy VL, Lee BC, Corbett JR, Ficaro EP. Variance estimation for myocardial blood flow by dynamic PET. IEEE Trans Med Imaging. 2015;34(11):2343-53.

12. Nesterov SV, Deshayes E, Sciagra R, Settimo L, Declerck JM, Pan XB, et al. Quantification of myocardial blood flow in absolute terms using (82)Rb PET imaging: the RUBY-10 study. JACC Cardiovasc Imaging. 2014;7(11):1119-27.

13. Cherry SR, Sorenson JA, Pherlps ME. Physics in nuclear medicine 4th ed. Philadelphia: Elsevier/Saunders; 2012.

14. Schwaiger M, Ziegler S, Nekolla SG. PET/CT: challenge for nuclear cardiology. J Nucl Med. 2005;46(10):1664-78. 
15. Tang J, Rahmim A, Lautamaki R, Lodge MA, Bengel FM, Tsui BM. Optimization of Rb-82 PET acquisition and reconstruction protocols for myocardial perfusion defect detection. Phys Med Biol. 2009;54(10):3161-71.

16. Karp JS, Surti S, Daube-Witherspoon ME, Muehllehner G. Benefit of time-of-flight in PET: experimental and clinical results. J Nucl Med. 2008;49(3):462-70.

17. Armstrong I, Tout $\mathrm{D}$, Tonge $\mathrm{C}$, Arumugam P. Time-of-flight reduces the severity of $\mathrm{CT}$ mis-registration artefacts in rubidium-82 cardiac PET. J Nucl Med. 2013;54:1636.

18. Mettivier G, Tabacchini V, Conti M, Russo P. Signal-to-noise gain at variable randoms ratio in TOF PET. IEEE Trans Nucl Sci. 2012;59(5):1948-57.

19. DiFilippo FP, Brunken RC. Impact of time-of-flight reconstruction on cardiac PET images of obese patients. Clin Nucl Med. 2017;42(2):e103-e8.

20. Conti M. Why is TOF PET reconstruction a more robust method in the presence of inconsistent data? Phys Med Biol. 2011;56(1):155-68.

21. Armstrong IS, Tonge CM, Arumugam P. Impact of point spread function modeling and time-of-flight on myocardial blood flow and myocardial flow reserve measurements for rubidium-82 cardiac PET. J Nucl Cardiol. 2014;21(3):467-74.

22. Rapisarda E, Bettinardi V, Thielemans K, Gilardi MC. Image-based point spread function implementation in a fully 3D OSEM reconstruction algorithm for PET. Phys Med Biol. 2010;55(14):4131-51.

23. Watson CC. New, faster, image-based scatter correction for 3D PET. IEEE Trans Nucl Sci. 2000;47(4):1587-94.

24. Zaidi H. Scatter modelling and correction strategies in fully 3-D PET. Nucl Med Commun. 2001;22(11):1181-4.

25. Polycarpou I, Marsden PK, Tsoumpas C. A comparative investigation of scatter correction in 3D PET. J Phys Conf Ser. 2011:317:012022.

26. Slomka PJ, Alessio AM, Germano G. How to reconstruct dynamic cardiac PET data? J Nucl Cardiol. 2017;24(1):291-3.

27. Hudson HM, Larkin RS. Accelerated image-reconstruction using ordered subsets of projection data. IEEE Trans Med Imaging. 1994;13(4):601-9.

28. Knesaurek K, Machac J, Krynyckyi BR, Almeida OD. Comparison of 2-dimensional and 3-dimensional Rb-82 myocardial perfusion PET imaging. J Nucl Med. 2003;44(8):1350-6.

29. Christian PE. Nuclear medicine and PET/CT: technology and techniques. 7th ed. Mosby/Elsevier: St. Louis Mo; 2012.

30. Martin CC, Christian BT, Satter MR, Nickerson LH, Nickles RJ. Quantitative PET with positron emitters that emit prompt gamma rays. IEEE Trans Med Imaging. 1995;14(4):681-7.

31. Esteves FP, Nye JA, Khan A, Folks RD, Halkar RK, Garcia EV, et al. Prompt-gamma compensation in Rb-82 myocardial perfusion 3D PET/CT. J Nucl Cardiol. 2010;17(2):247-53.

32. Dunet $\mathrm{V}$, Klein R, Allenbach G, Renaud J, deKemp RA, Prior JO. Myocardial blood flow quantification by Rb-82 cardiac PET/CT: a detailed reproducibility study between two semi-automatic analysis programs. J Nucl Cardiol. 2016;23(3):499-510.

33. Klein R, Renaud JM, Ziadi MC, Thorn SL, Adler A, Beanlands RS, et al. Intra- and inter-operator repeatability of myocardial blood flow and myocardial flow reserve measurements using rubidium-82 pet and a highly automated analysis program. J Nucl Cardiol. 2010;17(4):600-16.

34. Cerqueira MD, Weissman NJ, Dilsizian V, Jacobs AK, Kaul S, Laskey WK, et al. Standardized myocardial segmentation and nomenclature for tomographic imaging of the heart. A statement for healthcare professionals from the Cardiac Imaging Committee of the Council on Clinical Cardiology of the American Heart Association. Circulation. 2002;105(4):539-42.

35. Lin LI. A concordance correlation coefficient to evaluate reproducibility. Biometrics. 1989:45(1):255-68

36. Giavarina D. Understanding Bland Altman analysis. Biochem Med (Zagreb). 2015;25(2):141-51.

37. Presotto L, Gianolli L, Gilardi MC, Bettinardi V. Evaluation of image reconstruction algorithms encompassing time-of-flight and point spread function modelling for quantitative cardiac PET: phantom studies. J Nucl Cardiol. 2015;22(2):351-63.

38. Renaud JM, Yip K, Guimond J, Trottier M, Pibarot P, Turcotte E, et al. Characterization of 3-dimensional PET systems for accurate quantification of myocardial blood flow. J Nucl Med. 2017;58(1):103-9.

39. Morey AM, Kadrmas DJ. Effect of varying number of OSEM subsets on PET lesion detectability. J Nucl Med Technol. 2013:41(4):268-73.

40. Maebatake A, Akamatsu G, Miwa K, Tsutsui Y, Himuro K, Baba S, et al. Relationship between the image quality and noise-equivalent count in time-of-flight positron emission tomography. Ann Nucl Med. 2016;30(1):68-74.

41. Akamatsu G, Ishikawa K, Mitsumoto K, Taniguchi T, Ohya N, Baba S, et al. Improvement in PET/CT image quality with a combination of point-spread function and time-of-flight in relation to reconstruction parameters. J Nucl Med. 2012;53(11):1716-22.

\section{Submit your manuscript to a SpringerOpen ${ }^{\circ}$ journal and benefit from:}

- Convenient online submission

- Rigorous peer review

- Immediate publication on acceptance

- Open access: articles freely available online

- High visibility within the field

Retaining the copyright to your article

Submit your next manuscript at $\gg$ springeropen.com 